\title{
What Benford Can Tell Us About Cover Pools - An Empirical Analysis
}

\author{
Stephan Kienle, Darmstadt University of Technology, Germany
}

\begin{abstract}
Leading digits often follow a distribution described by Newcomb (1881) and Benford (1938). We apply this phenomenon known as Benford's Law on cover assets provided by issuers of German covered bonds. The main finding of the empirical analysis is that leading digits of these assets seem to follow the Benford distribution. Standard statistical evidence, however, might be misleading due to effects of large data sets. Consequently, the present paper also provides an example of how to deal with large data sets when a Benford distribution is assumed.
\end{abstract}

Keywords: Benford's Law; Pfandbriefe; Mean Absolute Deviation

\section{INTRODUCTION}

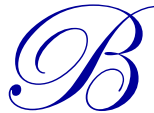

enford's Law is based on the observation that leading digits rarely follow a uniform distribution, but are rather distributed logarithmically. In contrast to many people's intuition, figures starting with lower integers are far more frequently observable than figures with higher integers as leading digits. Under Benford, the probability of a 1 as first digit is $30.1 \%$ and decreases monotonously for the other digits. Eventually, a 9 is much less likely as first digit and only observable in $4.6 \%$ of the cases. This regularity was first described by Newcomb (1881) and later rediscovered by Benford (1938). Accordingly, the probability for the first significant digit is given as

$$
P\left(D_{1}=d_{1}\right)=\log _{10}\left(1+d_{1}^{-1}\right)
$$

with $d_{1} \in\{1,2, \ldots 9\}$.

Later on, Benford's Law has become an important tool to detect possible manipulations in balance sheets and tax returns. This application was especially promoted by Nigrini (1996a, 1996b, 1999). Besides, it was used for the identification of human interactions on figures in the context of empirical research (Diekmann 2007, Toedter, 2009, among others).

In the present paper, we apply the regularity on asset data provided for German covered bonds (so-called Pfandbriefe). We focus on mortgage Pfandbriefe, i.e. bonds which are collateralized by residential and commercial mortgage loans. Our goal is to find out whether the distribution is observable and might be used to identify human interactions.

A graphical inspection reveals a Benford-like pattern. Due to the high number of single digits in the overall sample set, standard $\mathrm{Chi}^{2}$ tests however fail to significantly reveal a Benford distribution at a common confidence level. To overcome these issues, we apply additional test procedures. First, we use an approach suggested by Drake and Nigrini (2000) based on mean absolute deviations. Second, we utilize a mean test described by Toedter (2009). Both tests have in common that they are less sensitive to the sample size and might therefore be appropriate keeping in mind the high number of first digits under consideration.

The remainder of the paper is organized as follows. The next section gives an overview of the wide strand of literature dealing with applications of Benford's Law and outlines the methodologies applied in the paper at hand. In section 3 we provide an empirical analysis of the collateral pools of German covered Bonds and illustrate to 
which extent the leading digits conform with the expected distribution. The last section concludes by summarizing the key results, i.e. that the cover pools are supposed to follow Benford's Law.

\section{RESEARCH FRAMEWORK}

\section{Related Literature}

The distribution which is nowadays known as Benford's Law has first been described by Newcomb (1881) who has realized that in logarithmic books pages with lower digits were more worn out than pages with higher digits. He drew the conclusion that numbers tend to begin with lower digits more frequently. Apparently unaware of Necomb's findings half a century before, the same pattern was rediscovered by Benford (1938) who also provided empirical evidence supporting his hypothesis that this distribution is widely spread by testing the first digit frequencies of a large number of figures drawn from different sources.

Later, Hill (1995) provided a proof for Benford's law and showed in which cases a set of numbers conforms to Benford. He stated that "if distributions are selected randomly (in any "unbiased" way) and random samples are then taken from each of these distributions", the resulting data will converge to Benford even if the single distributions do not follow the law individually.

There exist several criteria to decide whether a set of numbers may conform to Benford. Qualitative factors are for instance described in Durtschi et al. (2004). Hence, the figures shall comprise a large, unrestricted data set which is not influenced by human thought. For instance, prices which are set below psychological barriers do usually not comply with Benford. Wallace (2002) suggested two quantitative measures to assess whether a distribution may be expected to obey Benford's Law. First, the mean of the observed digits shall be larger than the median. Second, the distribution shall exhibit a positive skewness.

Nowadays, Benford's Law has been applied to auditing and accounting. Based on the assumption, that people who fabricate figures probably distribute figures uniformly, Varian (1972) suggested the use of the law as a fraud indicator. Against this background, Carslaw (1988) and Thomas (1989) detected earning manipulations for New Zealand and US firms respectively. Nigrini (1996a, 1996b, 1999) focused on fraud detection by applying the law on American tax declarations. His findings indicated that people tend to understate their income to reduce taxation. Quick and Wolz (2003) for instance applied Benford's Law to financial statements of German companies and found that these figures are in line with the expected distribution. Drake and Nigrini (2000) and Durtschi et al. (2004) provided operational descriptions how to implement Benford's law for auditing purposes.

In the meantime, there are several papers applying the anomaly to other areas of interest. Rauch et al. (2011) used the distribution on macroeconomic data to identify probably fraudulent data from the Greek government before joining the euro area. Marchi and Hamilton (2006) studied environmental law compliance by examining discrepancies in air pollution reports. Diekmann (2007) and Toedter (2009) present an application on scientific data. While Diekmann (2007) discovered that estimates drawn from four volumes of the American Journal of Sociology overwhelmingly did not follow Benford, Toedter (2009) investigated empirical research and forecast data. He found that the relative frequencies of the first digits are broadly in line with Benford, whereas second digits of Consensus Forecasts show an excess of zeros and fives.

\section{Applied Methodology}

One of the most popular test procedures is the application of a standard $\mathrm{Chi}^{2}$ test (see for instance Cleary and Thibodeau 2005). The test statistics for the leading digit is defined as

$$
C H I^{2}=N \sum_{d 1=1}^{9} \frac{\left(h_{d 1}-p_{d 1}\right)^{2}}{p_{d 1}}
$$

where $\mathrm{h}_{\mathrm{d} 1}$ and $\mathrm{p}_{\mathrm{d} 1}$ are the observed and the expected proportion of digit frequency. The null hypothesis of being Benford distributed is rejected if $\mathrm{CHI}^{2}>\chi_{\alpha}^{2}(8)$ at a chosen level of significance $\alpha$. However, the $\mathrm{Chi}^{2}$ test is often 
unsuitable especially for larger sample sizes as it is sensitive to the number of observations (see for example Morrow 2010 and Nigrini 2000) and the null hypothesis of obeying Benford's Law will eventually be rejected with increasing sample size for a given significance level. To overcome this excess power problem two alternative test statistics are presented which seem more appropriate to deal with large data sets. First, we test the goodness-of-fit by calculating the mean absolute deviation (MAD) which is defined as

$$
M A D=\frac{1}{9} \sum_{d 1=1}^{9}\left|h_{d 1-} p_{d 1}\right|
$$

In contrast to the $\chi^{2}$-test there are no clear cut-off values. However, according to Drake and Nigrini (2000), the following bandwidths in table 1 can be applied to assess whether conformity is given.

Table 1. MAD conformity bandwidths

\begin{tabular}{lc}
\hline Close & $0.000-0004$ \\
Acceptable & $0.004-0008$ \\
Marginally acceptable & $0.008-0012$ \\
Nonconformity & $>0.012$ \\
\hline
\end{tabular}

Source: Drake and Nigrini (2000).

Second, we apply the mean test by Toedter (2009), which relates the observed mean of the digits to the mean $\mu(\mathrm{d} 1)$ and variation $\sigma^{2}(\mathrm{~d} 1)$ under Benford's law as follows

$$
\begin{aligned}
& T_{d 1}=\sqrt{N} \frac{\sum_{d 1=1}^{9}(d 1+0,5) h_{d 1}-\mu(d 1)}{\sqrt{\sigma^{2}(d 1)}} \\
& \text { with } \mu(d 1)=\sum_{d 1=1}^{9} p_{d 1} * d 1 \text { and } \sigma^{2}(d 1)=\sum_{d 1=1}^{9}(d 1-\mu(d 1))^{2} * p_{d 1} .
\end{aligned}
$$

In contrast to the test proposed by Drake and Nigrini (2000), the mean test allows for clear cut-off levels as it is approximately standard normally distributed.

\section{EMPIRICAL ANALYSIS}

\section{Data Set}

Pfandbriefe are issued by credit institutions as collateralized interest-bearing bonds. Depending on the type of assets to secure these payments, they are referred to as Mortgage Pfandbriefe, Ship Pfandbriefe, Aircraft Pfandbriefe or Public Pfandbriefe. All cover assets are recorded in a cover register and belong to the respective cover pool. In what follows, we rely on Mortgage Pfandbriefe that are usually covered by various single loans. The analysis is based on data provided by the Association of German Pfandbrief Banks (vdp), an umbrella organization which comprises basically all issuer of legally defined German Covered Bonds. Our data set is made up of figures from the fourth quarter 2008 to the fourth quarter of 2014 for 39 different credit institutions. Hence, the analysis covers approximately 25 thousand significant leading digits. The data set seem to allow the assumption of a Benford distribution as it is a large and unrestricted one based on a combination of different distributions, for instance stock of real estate multiplied by real estate prices. Furthermore, the measures proposed by Wallace (2002) also support this assumption as illustrated in table 2, i.e. the mean of the data exceeding its median and the skewness is positive.

Table 2. Data set measures

\begin{tabular}{ll}
\hline Mean & 3.4 \\
Median & 3.0 \\
Skewness & 0.7 \\
\hline
\end{tabular}

Source: Own calculation.

\section{Testing Benford}

Following Cleary and Thibodeau (2005) we focus on the significant first digit distribution by providing a $\mathrm{Chi}^{2}$ and mean tests for the cover pool assets. As described by Baryla (2012), the first significant figure is the first non-zero digit of a number starting from the left side. Figure 1 depicts that the empirical distribution is by and large 
rather similar to the theoretical one suggested by Benford even though integer 1 is more often observed than expected while in particular a 2 and a 9 is not observed as frequently as implied by Benford.

Figure 1. First digit distribution for cover pools (Q4.2008 - Q1.2014)

$\mathrm{P}(\mathrm{d} 1)$

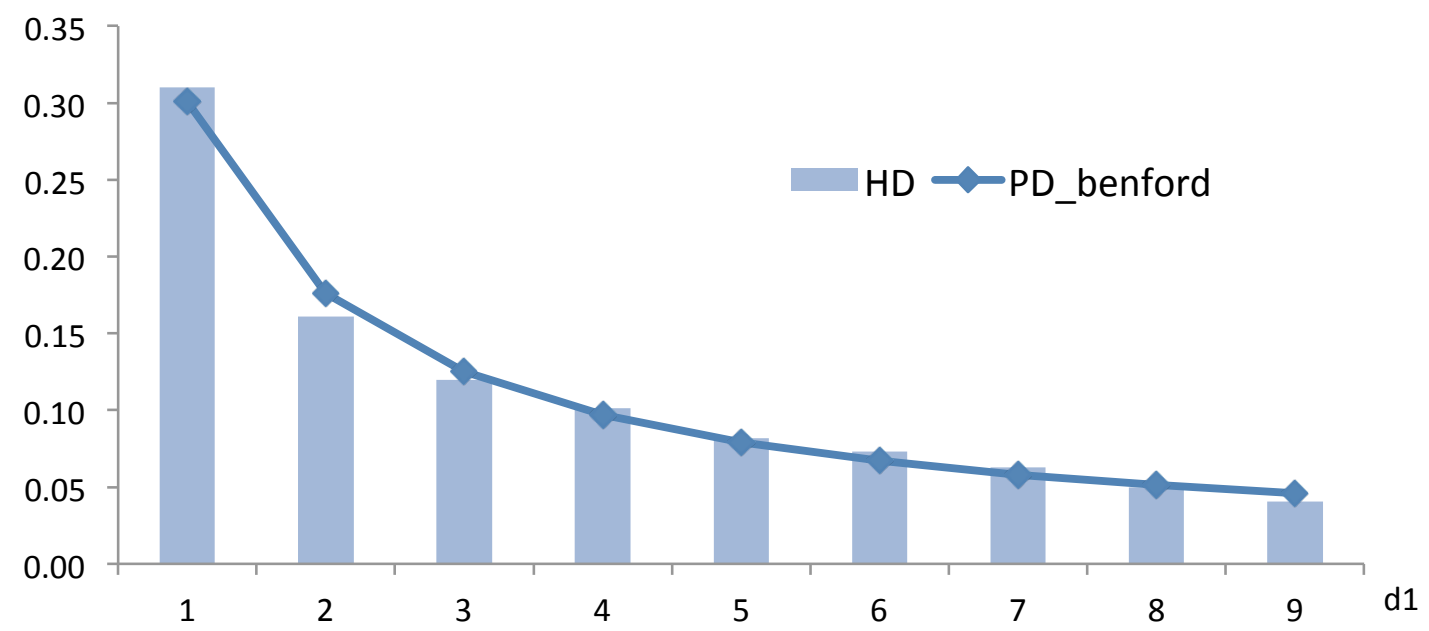

To gain further insights, we first apply a standard $\mathrm{Chi}^{2}$ test. For the overall sample, the $\mathrm{Chi}^{2}$ test clearly rejects the null of a Benford distribution. This seems reasonable, due to the sample size and the characteristic of the test statistic which tends to reject the null as a type II error becomes less likely for large data sets. The null of a Benford distribution is also rejected on a yearly basis at common confidence levels. However, the rejection is not that clear for single years. Conscious of the size of the data set, we also apply both MAD and mean test which is dedicated to be applied on large data sets. In both cases, for the overall sample as well as the individual subsamples, the null of being Benford distributed cannot be rejected. The results are summarized in table 3 .

Table 3. Yearly and overall distribution of cover assets

\begin{tabular}{lccccccc}
\hline \multicolumn{1}{r}{ Year } & $\mathbf{2 0 0 9}$ & $\mathbf{2 0 1 0}$ & $\mathbf{2 0 1 1}$ & $\mathbf{2 0 1 2}$ & $\mathbf{2 0 1 3}$ & $\mathbf{2 0 1 4}$ & Overall \\
\hline $\mathrm{N}$ & 3,311 & 3,584 & 3,894 & 4.391 & 4,352 & 3,132 & 23,787 \\
$\mathrm{Chi}^{2}$ & 36.1 & 16.5 & 16.8 & 44.6 & 36.7 & 14.7 & 87.7 \\
$\mathrm{MAD}$ & 0.010 & 0.006 & 0.006 & 0.008 & 0.005 & 0.004 & 0.006 \\
Mean & 1.12 & 0.80 & 0.96 & 0.38 & 0.16 & 1.53 & 1.03 \\
\hline
\end{tabular}

Source: Data drawn from vdp, own calculations. Overall data cover first digits from the fourth quarter 2008 to the fourth quarter of 2014.

\section{CONCLUSION}

To our knowledge, this is the first paper to investigate whether Benford's Law holds for the cover assets of German covered bonds. In our analysis, we focus on mortgage Pfandbriefe, i.e. bonds secured by individual commercial and residential loans.

Taking the sample size explicitly into consideration, we provide some additional test statistics based on MAD and mean tests which are dedicated to be applied on large data sets. By doing so we provide some evidence for the hypothesis that Benford's Law is applicable on the cover assets. Furthermore, we provide an example for how standard $\mathrm{Chi}^{2}$ test might be misleading in the presence of large sample sizes. We therefore contribute to the mitigation of potentials shortcomings of the test. 


\section{AUTHOR INFORMATION}

Stephan Kienle is a doctoral student at the Darmstadt University of Technology. He did his undergraduate studies in business sciences and economics at the University of Applied Sciences of the Deutsche Bundesbank and at the Goethe University at Frankfurt, where he finally graduated with a Master equivalent degree in economics. His main research interest is in macroeconomics with a special focus on monetary policy.

\section{AUTHOR NOTE}

The views expressed in this paper are those of the author alone. Any remaining shortcomings are entirely the responsibility of the author.

\section{REFERENCES}

Baryla, M. (2012); Some Remarks about Benford's Distribution, Mathematical Economics 8(15), 5-15.

Benford, F. (1938), The Law of Anomalous Numbers, Proceedings of the American Philosophical Society 78(4), 551-572.

Carslaw, C. A. P. N. (1988), Anomalies in income numbers: Evidence of goal oriented behavior, The Accounting Review LXIII (2), 321-327.

Cleary, R., and Thibodeau, J. C. (2005), Applying Digital Analysis Using Benford's Law to Detect Fraud: The Dangers of Type I Errors, Auditing: A Journal of Practice \& Theory 24 (1), 77-81.

Drake, P. D., and Nigrini, M. J. (2000), Computer assisted analytical procedures using Benford's Law, Journal of Accounting Education 18(2), 127-146.

Durtschi, C., Hillison, W. and Pacini, C. (2004), The Effective Use of Benford's Law to Assist in Detecting Fraud in Accounting Data, Journal of Forensic Accounting $V$, 17-34.

Hill, T.P. (1995), A Statistical Derivation of the Significant Digit Law, Statistical Science 10(4), 354-363.

Marchi, S. and Hamilton, J. T. (2006), Assessing the Accuracy of Self-Reported Data: an Evaluation of the Toxics Release Inventory, Journal of Risk and Uncertainty 32(1), 57-76.

Morrow, J. (2014), Benford's Law, Families of Distributions and a Test Basis, CEP Discussion Paper 1291.

Newcomb, S. (1881), Note on the Frequency of Use of the Different Digits in Natural Numbers, American Journal of Mathematics 4(1), 39-40.

Nigrini, M. J. (1996), Taxpayer Compliance Application of Benford's Law, Journal of the American Taxation Association 18(1), 72-92.

Nigrini, M. J. (1996), Added Value with Digital Analysis, The Internal Auditor 56(1), 21-23.

Nigrini, M. J. (2000), Digital Analysis Using Benford's Law, Global Audit Publications, Vancouver, Canada.

Nigrini, M. J. (1999), I've Got Your Number, Journal of Accountancy 187(5), 79-84.

Quick R, Wolz M (2003), Benford's law in deutschen Rechnungslegungsdaten, Betriebswirtschaftliche Forschung und Praxis 55, 208-224.

Rauch, B, Göttsche, M., Brähler, G., Engel, S. (2012), Fact and Fiction in EU-Governmental Economic Data, German Economic Review 12(3), 243-255.

Thomas, J. K. (1989), Unusual patterns in reported earnings, The Accounting Review LXIV(4), 773-787.

Toedter, K. (2009), Benford's Law as an indicator of fraud in economics, German Economic Review 10(3), 339-351.

Varian, H. R. (1972), Benford's law, The American Statistician 26(3), 65-66.

Wallace, W. A. (2002), Assessing the quality of data used for benchmarking and decisionmaking, The Journal of Government Financial Management 51 (3), 16-22. 


\section{NOTES}

